\title{
Interrelationships between Rheological, Morphological, and Mechanical Properties of Polystyrene/Liquid Crystalline Polymer Blends
}

\author{
Gi Dae Chol, Seung Hyun KIm, and Won $\mathrm{Ho} \mathrm{Jo}^{\dagger}$ \\ Department of Fiber and Polymer Science, Seoul National University, Seoul 151-742, Korea
}

(Received November 20, 1995)

\begin{abstract}
The effects of the viscosity ratio of dispersed phase to matrix on the rheological, morphological, and mechanical properties of blends of polystyrene and two rheologically different liquid crystalline polymers (LCPs) were investigated. Two different rheological behaviors are obtained by blending polystyrene (PS) and two different LCPs: one is the case that the viscosity of dispersed phase is lower than that of matrix phase as observed in PS/Rodrun blends, and another one is the case that the viscosity of dispersed phase is higher than that of matrix phase as observed in PS/Vectra blends. Scanning electron micrographs of fracture surfaces of injection-molded samples show that the finely distributed LCP fibril structure is observed for PS/Rodrun blends where the viscosity of the dispersed LCP phase is lower than that of the polystyrene matrix phase, whereas dispersed LCP phase in PS/Vectra blends show the spherical form of LCP domains. Mechanical properties of injection molded specimens show that the modulus of PS/Rodrun blends shows a strong positive deviation from the simple additive rule, whereas the modulus of PS/Vectra blends follows the simple additive rule of mixture. These mechanical properties are well consistent with the morphological characteristics of the PS/LCP blends.
\end{abstract}

KEY WORDS Polystyrene / Liquid Crystalline Polymers / Viscosity Ratio / Rheology / Morphology / Mechanical Properties/

The concepts of tailoring polymer properties through blending with other polymers and fillers have long been recognized as a viable method to meet specific applications. But, most polymer pairs are immiscible and form multiphase domains on mixing. The morphology of immiscible polymer blends depend on a multitude of factors including the viscosity ratio, composition, interfacial tension, magnitude and type of flow field, and mixing time, etc. ${ }^{1,2}$

In particular, polymer blends containing liquid crystalline polymers (LCPs) have been studied intensively to achieve a so-called in situ composite structure with oriented LCP fibrils in the thermoplastic matrix. ${ }^{3-12}$ In most cases the addition of LCP increases the mechanical strength and the stiffness of the thermoplastic matrix polymer. Moreover, even a relatively small amount of LCP may induce a reduction in the melt viscosity, and thus make the processing easier. The LCP may also improve other properties of thermoplastics, such as dimensional stability and thermal stability. ${ }^{13,14}$ When the LCP is a minor component, it is potentially capable of forming highly elongated fibrous structures parallel to the extensional flow direction. The oriented LCPs in the matrix not only improve the mechanical properties of thermoplastic materials, but also make the processing free from problems associated with conventional fiber reinforced composites such as fiber breakage, abrasions of the processing machinery, increase in viscosity, energy consumption, and difficulties in uniform dispersion of fibers. ${ }^{15,16}$

The resultant properties of LCP blends depend on the amount and properties of the individual polymeric components as well as the morphology of the dispersed LCP domains, and the interfacial adhesion between the phases. ${ }^{17-19}$ The primary factors determining the morphologies of the LCP domains in the thermoplastic

\footnotetext{
$\uparrow$ To whom correspondence should be addressed.
}

matrix are the LCP content, processing conditions, and rheological characteristics of the blend components such as the viscosity ratio. ${ }^{20,21}$ The morphology is also affected by the interaction between the phases, which may be modified by the introduction of suitable compatibilizers. $^{22,23}$

The effect of viscosity ratio on the morphology of immiscible polymer blends has been studied by several researchers. Min et al $^{24}$ found for polyethylene/polystyrene (PE/PS) blends that, when the dispersed phase had a lower viscosity, the dispersed phase formed long fibers in the matrix, but with higher viscosity the dispersed phase was in the form of discrete droplets. Crevccoeur and Groeninckx ${ }^{11}$ have studied blends of PS and LCP and reported that the LCP formed fine fibrils in PS matrix and that the modulus increased with the LCP volume fraction. They indicated that in order to elongate the dispersed LCP into fibrils, an elongational flow is important and necessary. Basett and $\mathrm{Yee}^{25}$ investigated processing methods that produce fibers reinforced by in-situ formed fibrils. They reported that high modulus fibers were produced from PS/LCP blends when an extrusion die of infinite $\mathrm{L} / \mathrm{D}$ ratios was used.

Despite efforts related to the study of deformation of the dispersed LCP phase in polymer blends, only few attempts have been made to analyze the effect of viscosity ratio on the blend morphology and the mechanical properties. Moreover, no consistent systematic approach has been made that can satisfactorily describe the effects of the viscosity ratio on the interrelationships between rheological, morphological, and mechanical properties of blends containing LCP. Thus we examined systematically the effects of the viscosity ratio of dispersed phase to matrix phase on the rheological, morphological, and mechanical properties of LCP blends by using PS and two rheologically different LCPs. In this study, polystyrene has been chosen as a continuous matrix phase, and Vectra A950 or Rodrun LC5000 liquid crystalline 
copolyesters have been chosen as a dispersed phase. Here, the viscosity of two LCPs is largely different so that different levels of the viscosity ratio of dispersed phase to matrix are obtained.

\section{EXPERIMENTAL}

\section{Materials}

Two different LCPs are used in this study. One is a fully aromatic main chain copolyester and commercially known as Vectra A950 which is believed to be a random copolyester containing $75 / \mathrm{mol} \%$-hydroxybenzoic acid (HBA) and $25 \mathrm{~mol} \%$ 2,6-hydroxy naphthoic acid (HNA). Another is a semi-flexible main chain LCP and is commercially known as Rodrun LC5000 which is believed to be a copolyester containing $60 \mathrm{~mol} \% \mathrm{HBA}$ and $40 \mathrm{~mol} \%$ poly(ethylene terephthalate) (PET). The commercial grade polystyrene (PS) is used as a thermoplastic matrix polymer without further purification. The characteristics of polymers used in this study are reported in Table I.

\section{Blend Preparation}

All the polymer samples were completely dried under vacuum at $90^{\circ} \mathrm{C}$ for 24 hours before blending. The blends were prepared in the melt state using Mini-Max molder (CS-MMX, Custom Scientific Instruments, Inc.) at $295^{\circ} \mathrm{C}$. The melt mixing time was fixed at 5 minutes before injection to avoid degradation of the blend components. The amounts of LCP in blends were varied $10,20,30$, and $50 \%$ by weight.

\section{Thermal Analysis}

The thermal properties of all samples were measured using a differential scanning calorimeter (Perkin-Elmer DSC-7). Blend samples of $11-13 \mathrm{mg}$ were heated in a nitrogen atmosphere to $300^{\circ} \mathrm{C}$ at a heating rate of $20^{\circ} \mathrm{C}$ $\min ^{-1}$ and then cooled to room temperature. They were then reheated at a heating rate of $20^{\circ} \mathrm{Cmin}^{-1}$, and only the second running data were accepted to ensure that the thermal history of the different blends was identical. Before all DSC measurements the instrument was calibrated using standard materials such as indium and zinc.

\section{Morphological Observation}

The morphology of injection molded dumbbell type specimens was investigated using a scanning electron microscopy (SEM, JEOL, JSM-35). The samples were fractured perpendicular to the machine flow direction at the liquid nitrogen temperature. The cryogenically fractured surface was coated with gold sputtering unit and observed by SEM at an accelerating voltage of $25 \mathrm{kV}$.

Table I. Characteristics of polymer samples used in this study

\begin{tabular}{llcc}
\hline Sample & Source & $T_{\mathrm{g}} /{ }^{\circ} \mathrm{C}^{\mathrm{a}}$ & $T_{\mathrm{m}} /{ }^{\circ} \mathrm{C}^{\mathrm{a}}$ \\
\hline Vectra A950 & Hoechst Celanese Co. & - & $278^{\mathrm{b}}$ \\
Rodrun LC5000 & Unitika Ltd. & - & $275^{\mathrm{b}}$ \\
Polystyrene & Cheil Industries & 94 & - \\
\hline
\end{tabular}

${ }^{\mathrm{a}}$ Determined by DSC. ${ }^{\mathrm{b}}$ Nematic transition temperature.

\section{Rheological Properties}

The rheological properties of component polymers and their blends were measured on a Rheometrics Mechanical Spectrometer (RMS 800) with cone and plate mode under a nitrogen purging condition. Dynamic experiments were performed in oscillatory shear at $10 \%$ strain and dimensions of $25 \mathrm{~mm}$ in diameter. The frequency sweeps were performed from $0.1 \mathrm{rads}^{-1}$ to $100 \mathrm{rads}^{-1}$ at $295^{\circ} \mathrm{C}$. For pure PS and two LCPs, cooling experiments were also carried out at the parallel plate mode with a gap size of 1.6 to $1.8 \mathrm{~mm}$ by monitoring the change of complex viscosity with the temperature as the polymers were cooled from $300^{\circ} \mathrm{C}$. A cooling rate of about $5^{\circ} \mathrm{C} \mathrm{min}^{-1}$ was used for all these experiments. An angular frequency of $6.28 \mathrm{rad} \mathrm{s}^{-1}$ and the same strain levels as frequency sweep experiments were used for all the cooling experiments.

\section{Mechanical Properties}

Completely dried blend samples were injection molded using Mini-Max molder. Figure 1 shows the schematic drawings of the mixing and injection molding operations of Mini-Max molder and the dimensions of molded dumbbell specimen. The dimensions of the dumbbell type test specimens were $9 \mathrm{~mm}$ in length and $1.6 \mathrm{~mm}$ in diameter. Mechanical properties were measured by using an Instron Tensile Tester (model 4202) at room temperature. The extensional cross-head speed was 0.5 $\mathrm{mm} \min ^{-1}$. All the results were reported as an average of at least five measurements. The standard deviations were within $10 \%$ of the reported values.

\section{RESULTS AND DISCUSSION}

\section{Thermal Properties}

The results of thermal analysis for PS, LCPs, and their blends are shown in Figure 2. These results show no shift in the glass transition temperature of the PS component and the nematic transition temperature of the LCP
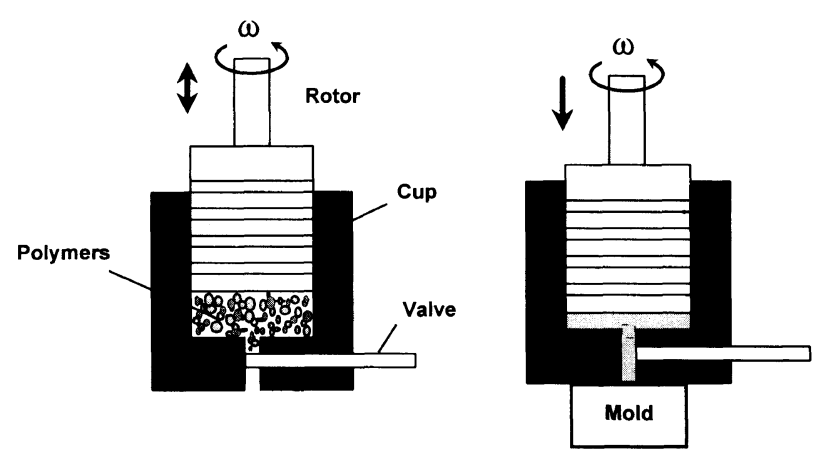

(a)

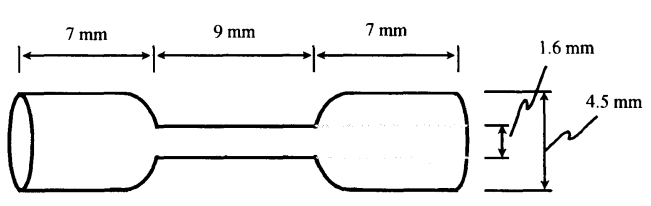

(b)

Figure 1. Schematic representation of melt mixing and injection molding operation of a Mini-Max injection molder (a) and molded tensile specimen (b). 

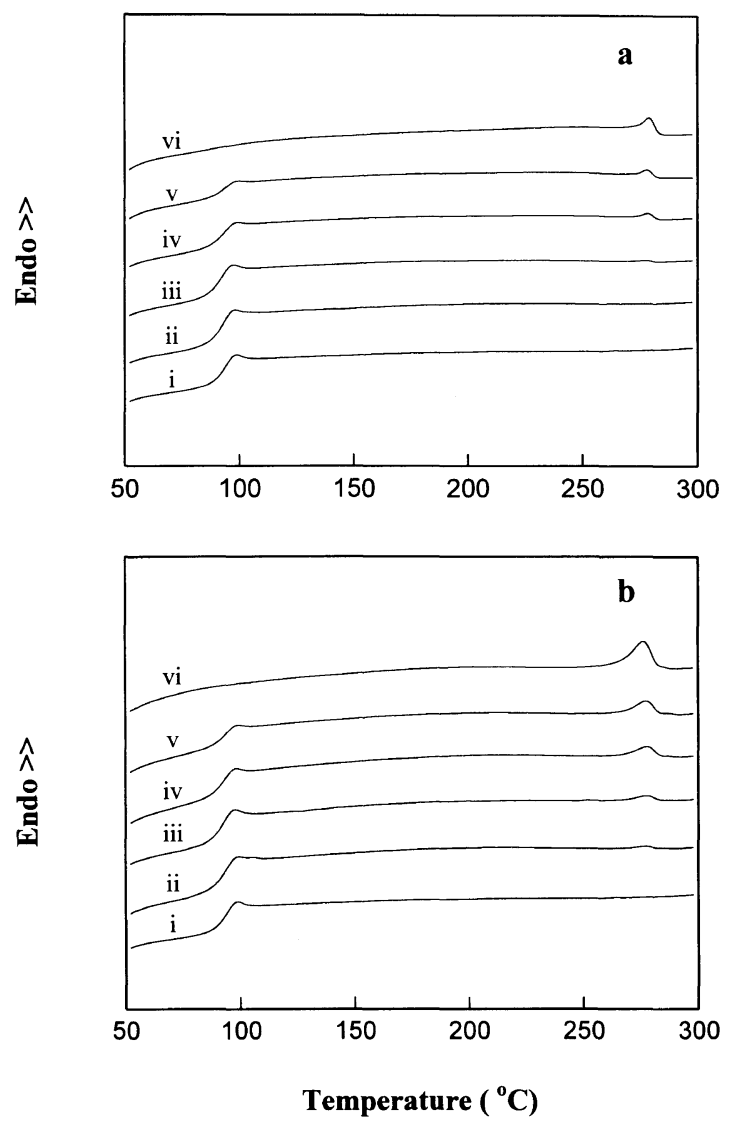

Figure 2. (a) DSC thermograms of PS/Vectra blends; (b) DSC thermograms of PS/Rodrun blends; (i) LCP 0; (ii) LCP 10; (iii) LCP 20; (iv) LCP 30; (v) LCP $50 \mathrm{wt} \%$; (vi) pure LCP.

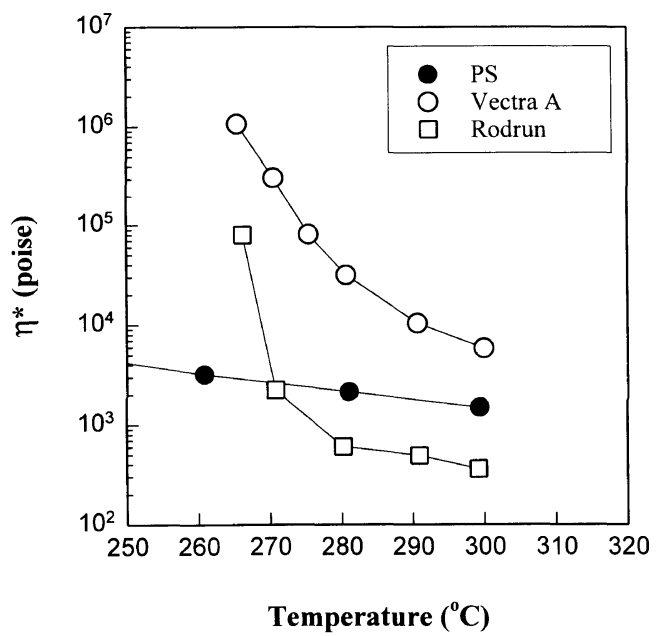

Figure 3. Complex viscosity as a function of temperature during cooling from $300^{\circ} \mathrm{C}$ for PS, Vectra, and Rodrun.

component in the blends with the blend ratio. Although the nematic transition of the LCP component is not visible clearly for blends with less than $30 \mathrm{wt} \%$ LCP for PS/Vectra blends, this is certainly attributable the relatively small heat of transition rather than to the miscibility. This independence of the transition temperatures on the entire blend composition clearly indicates an immiscibility of the two polymers. The results of the PS/Rodrun blends also shows an immiscibility similar to PS/Vectra blends.
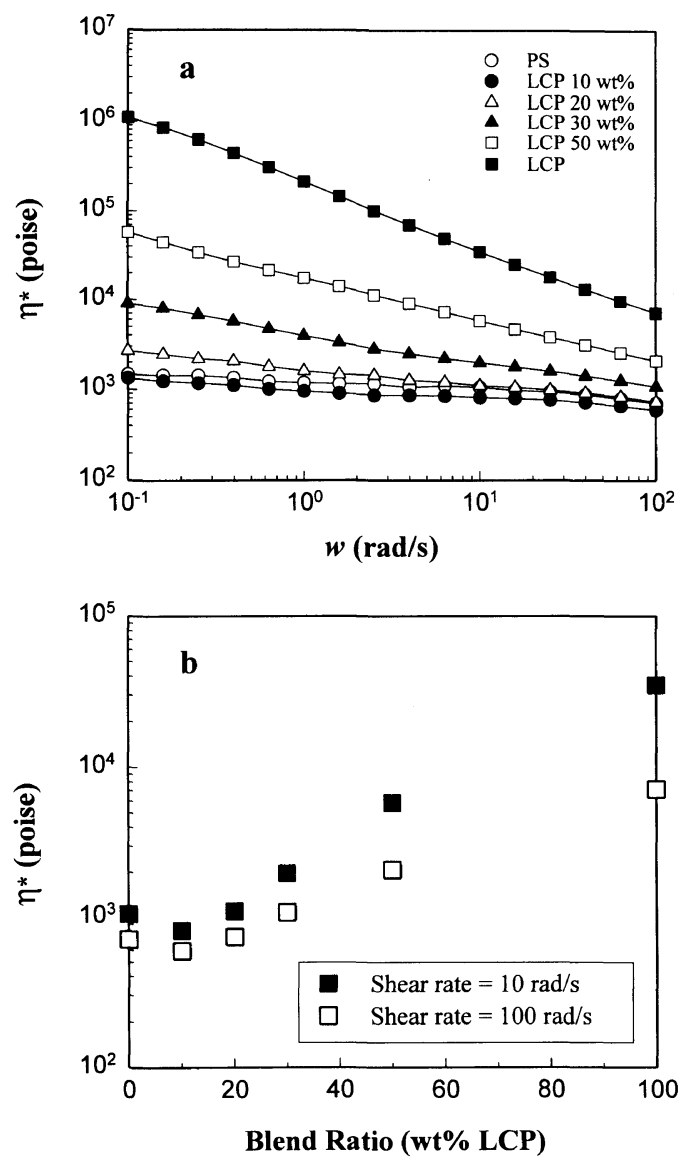

Figure 4. complex viscosity of PS/Vectra blends at $295^{\circ} \mathrm{C}$ as a function of (a) frequency and (b) LCP content.

\section{Rheological Properties}

In order to determine the temperature window within which a material may be processed, the samples were cooled from the melt. The resultant complex viscosities of pure PS, Vectra, and Rodrun LCP are shown as a function of temperature in Figure 3. The behavior in the cooling mode showed an important supercooling effect. The actual solidification temperature of LCP, judging by the rapid rise in complex viscosity, is somewhat lower than that of the melting temperature. This difference between solidification and melting temperature is often referred to as the "supercooling" window wherein the material is still viscous enough to flow. The complex viscosities of the pure PS are lower than those of the Vectra LCP, however, higher than Rodrun LCP in the processing temperature window of the blends. It is generally accepted that the lower viscosity of dispersed phase components in the matrix yields easier deformation in the blend by the flow fields. Therefore, PS/Rodrun blends have a higher potential to form the highly oriented structure of Rodrun LCP in the blend, whereas PS/Vectra blends have a difficulty to form the fibrillar dispersed LCP structure. The effect of the viscosity ratio on the morphology will be discussed in the following section.

The flow curves of PS, Vectra and their blends measured at $295^{\circ} \mathrm{C}$ are shown in Figure 4(a). The PS sample exhibits a slightly non-Newtonian behavior at the frequency range examined. This behavior is typical of thermoplastic polymers at the relatively low shear rate region. The pure LCP and blends containing high LCP 

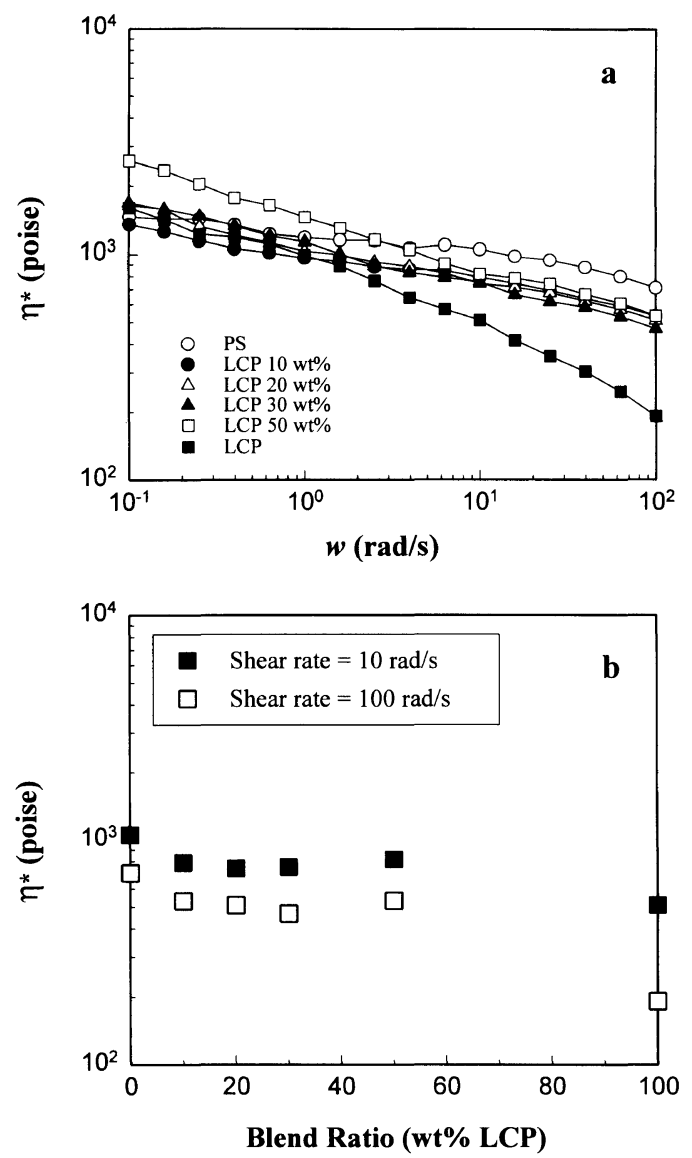

Figure 5. Complex viscosity of PS/Rodrun blends at $295^{\circ} \mathrm{C}$ as a function of (a) frequency and (b) LCP content.

content show a non-Newtonian behavior at the low frequency region and have lower power-law exponents. The viscosity as a function of the LCP content at two different frequencies is shown in Figure 4(b). The complex viscosities of PS/Vectra blends show a slight minimum in the viscosity at about $10 \mathrm{wt} \%$ LCP content. It was explained that the minimum in the viscosity-composition curves of the blends has been attributed to two main reasons: incompatibility between the two phases, and formation of elongated fibrils of the LCP phase which tend to lubricate the melt.

The flow curves of PS, Rodrun and their blends measured at $295^{\circ} \mathrm{C}$ are also shown in Figure 5(a). All compositions exhibit a more or less non-Newtonian behavior, and the viscosity decreases with the addition of a small content of LCP. The blends of high LCP concentration sample show a non-Newtonian behavior at low frequency and has lower power-law exponents. Especially, when $50 \mathrm{wt} \%$ of the LCP is added, a large increase of melt viscosity is observed at the low frequency. This typical shear thinning yield behavior may be related to the co-continuous polydomain structure of the LCP blend. In all cases except $50 \%$ LCP, the viscosity of the thermoplastic matrix is reduced by adding small amounts of the liquid crystal component, and the viscosity of the LCP blend lies between these of the two components (PS and LCP) as shown in Figure 5(b). This means that the lubrication effect of LCP reduces the viscosity of the blend when the viscosity of LCP (Rodrun) is lower than that of thermoplastic.
Two different rheological behaviors are observed for the blends of PS and two different LCPs. As shown in Figure 4(b), when the viscosity of the dispersed LCP (Vectra) is higher than that of the thermoplastic matrix polymer (PS), the viscosity-composition curve shows a minimum at about $10 \mathrm{wt} \%$ of the LCP content. When the viscosity of LCP (Rodrun) is lower than that of the thermoplastic matrix, the blend viscosity lies between those of the two components as shown in PS/Rodrun blends (Figure 5(b)). More recently, Mantia and Valenza $^{26}$ proposed a new mechanism for the prediction of a minimum in the viscosity-composition curves of LCP blends. They suggested that incompatibility and fibrillation are responsible for the reduction of the viscosity in the LCP based blends and that the particular viscosity ratio determining the phase morphology is also responsible for the presence of minima in these blends.

\section{Morphology}

Figure 6 shows the SEM photographs for fracture surfaces of injection molded dumbbell specimens of PS/Rodrun blends containing 10 and $20 \mathrm{wt} \%$ Rodrun LCP. As can be seen, the LCP particles change from coarse spheres and ellipsoids in the core region to fibrillar structure in the skin region. These morphologies can be explained by considering flow phenomena during the injection molding as follows. In the injection molding of LCP blends, a unique layered structure is formed due to the expanding radial flow in the vicinity of the gate, the "fountain flow" phenomena at the advancing melt front, and the non-isothermal shear flow that takes place when a hot melt flows into a cold cavity. The resultant morphology is almost a perfect alignment in the outer skin layer in the flow direction, and transverse orientation in the core region provided the mold cross-section is wide enough.

In the injection molding of tensile test specimens, three main mechanisms determine the LCP orientation in blends. First is the fountain flow which affects mainly the outer skin area, second is the converging type flow that develops when the blend flows from the tab of the tensile specimen to its narrow neck region. This elongation type flow gives rise to an alignment of the LCP phase in the flow direction. Finally, the shear flow mostly dominates the core region with its limited influence on LCP orientation. As seen in Figure 6, fibrillar alignment is a manifestation of the fountain flow effect. Furthermore, the injection molded tensile specimen shows some elongated particles also in the core region. This may be attributed to the converging type flow realized upon flow from the specimen's tab to its narrow neck section.

The SEM photographs of the fracture surfaces of PS/LCP blends with two different LCPs were shown in Figure 7. It should be emphasized that the LCP domains in the PS/Rodrun constitute of smaller diameter and longer fibers as compared to those in the PS/Vectra blends. From a comparison of micrographs of all blend compositions, it appeared that the fibril formation in PS/Rodrun blends was generally better than in PS/ Vectra. As discussed above, the lower viscosity of dispersed phase in the matrix results in easier deformation in the blend by the flow fields as observed in the PS/ 


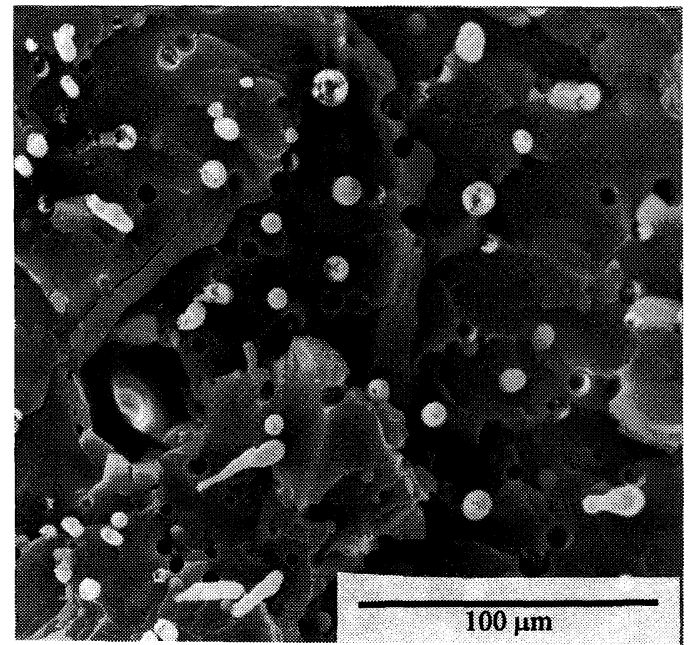

(a)

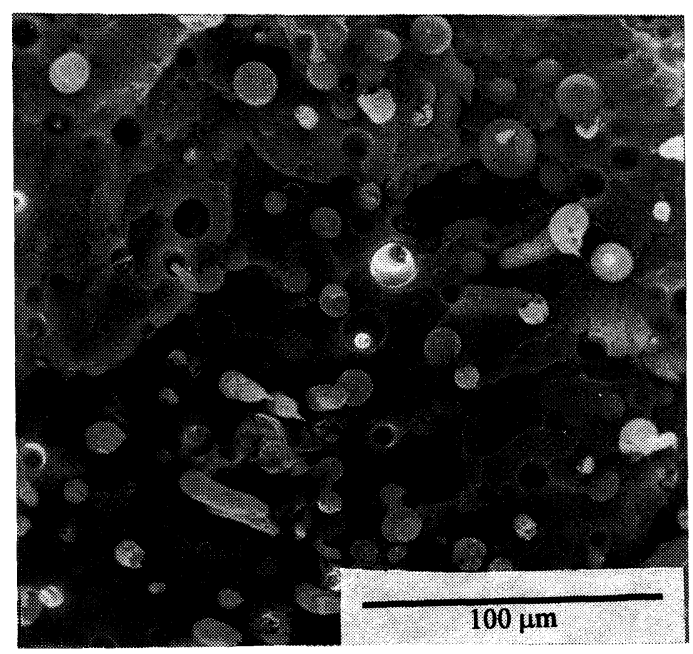

(c)

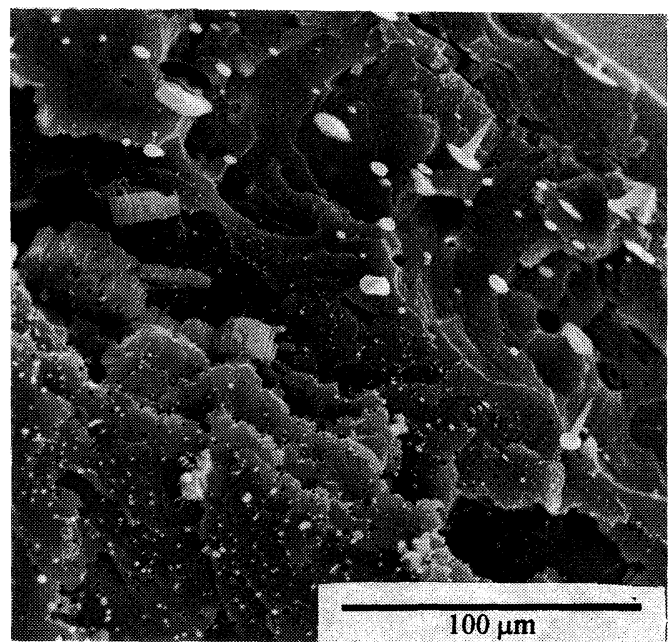

(b)

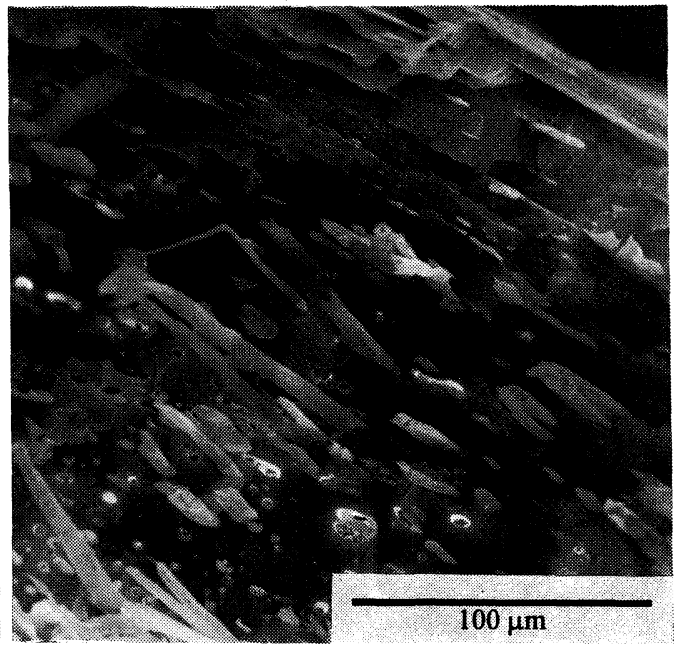

(d)

Figure 6. SEM photographs of the injection molded Rodrun/PS blends: (a) core region of LCP $10 \mathrm{wt} \%$ blend; (b) skin region of LCP $10 \mathrm{wt} \%$ blend; (c) core region of LCP $20 \mathrm{wt} \%$ blend; (d) skin region of LCP $20 \mathrm{wt} \%$ blend.

Rodrun blends. In other words, the morphology of the injection molded blends was strongly influenced by the viscosity ratio of LCP (Vectra or Rodrun) to thermoplastic matrix (PS). The finely and evenly distributed LCP structure was achieved when the viscosity of the dispersed LCP was lower than PS as in the case of PS/ Rodrun blends (Figures 7(b-1)-7(b-3)). But, when the viscosity of the dispersed LCP was higher than that of PS as in the case of PS/Vectra blends (Figures 7(a-1) 7(a-3)), the LCP domains become spherical cluster-like.

For all the LCP compositions, Rodrun LCP domains show the fine fibrillar structures in the blends, and they also appear to be distributed uniformly in PS matrix, whereas dispersed Vectra domains in the PS/Vectra blends exhibit less oriented or in the spherical form.

\section{Mechanical Properties}

Generally the mechanical properties of injection molded tensile specimens are expected to depend mainly on the LCP oriented fibrils in the flow direction. Tensile properties of pure components and their blends have been measured on injection molded specimens along the machine flow direction. The difference between the physical properties of the PS/Vectra and PS/Rodrun blends will be closely related to their respective blend morphologies. As discussed above, the fountain flow and the associated skin layer results in fibrillar LCP domains oriented in the machine direction. Moreover, the converging elongational flow from the tab of tensile specimens to its neck results in orientation development of the LCP in the testing direction.

Figure 8(a) shows tensile strength as a function of LCP composition for PS/LCP blends. The tensile strength of PS/Rodrun blends follows the simple additive rule of mixtures, whereas the tensile strength of PS/Vectra blends shows a negative derivation from the additivity rule. It is interesting to note that the tensile strengths of PS/Rodrun blends are higher than those of PS/Vectra blends although the pure Vectra shows higher tensile strength than the pure Rodrun LCP. This is attributable to better fibril formation of the LCP in the blends in the case of PS/Rodrun blends, as shown in Figure 7. Also the interfacial adhesion between the dispersed LCP fibrils and the PS thermoplastic is probably greater in the case 

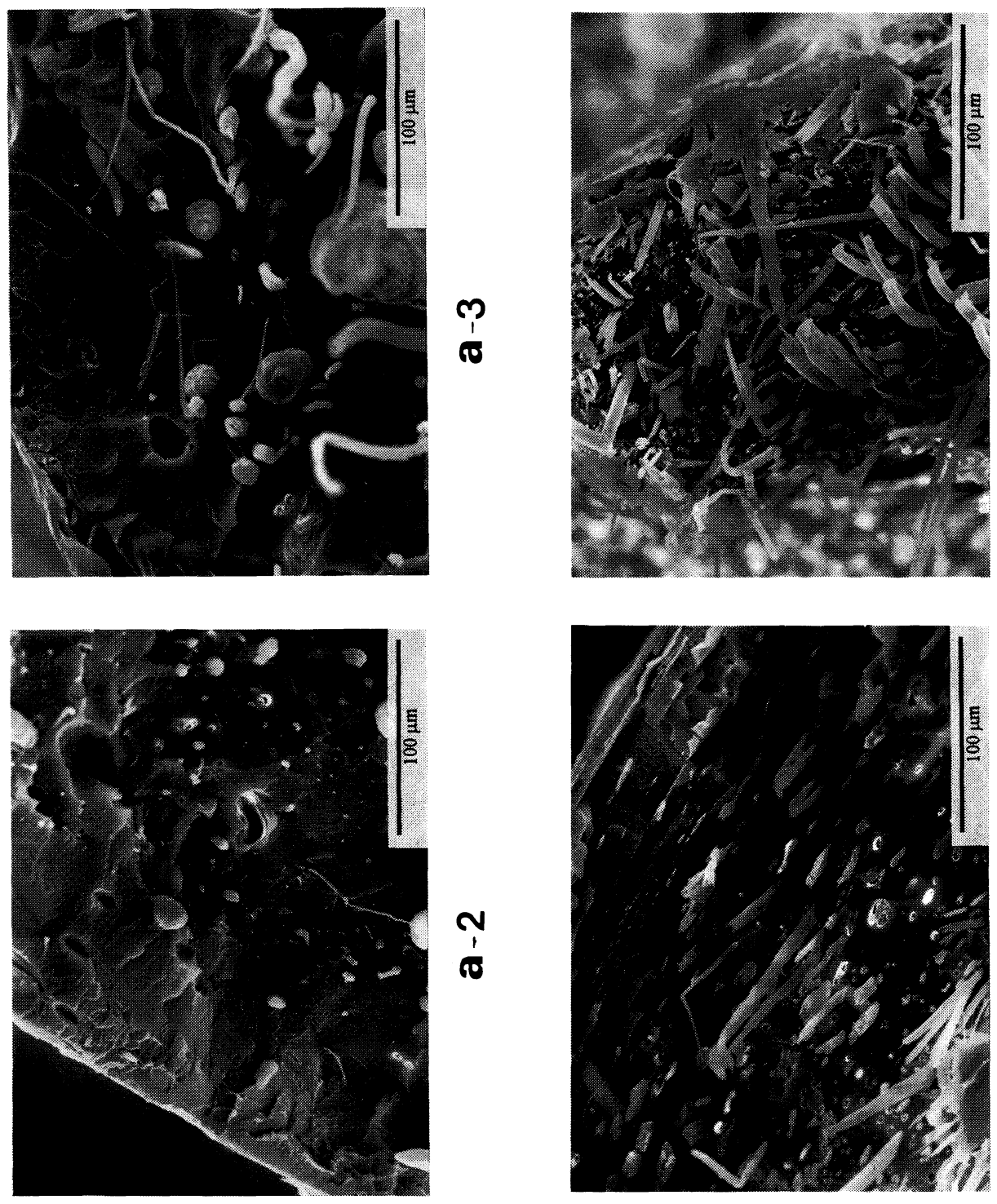

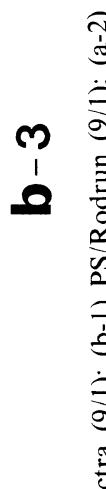

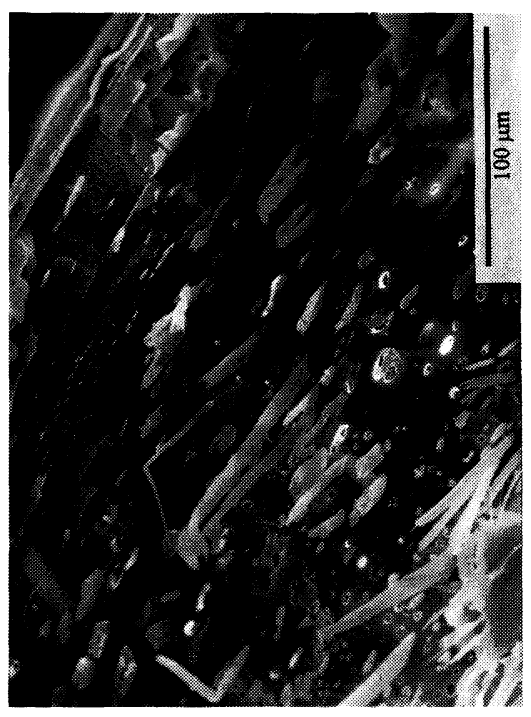

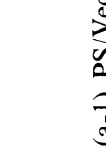
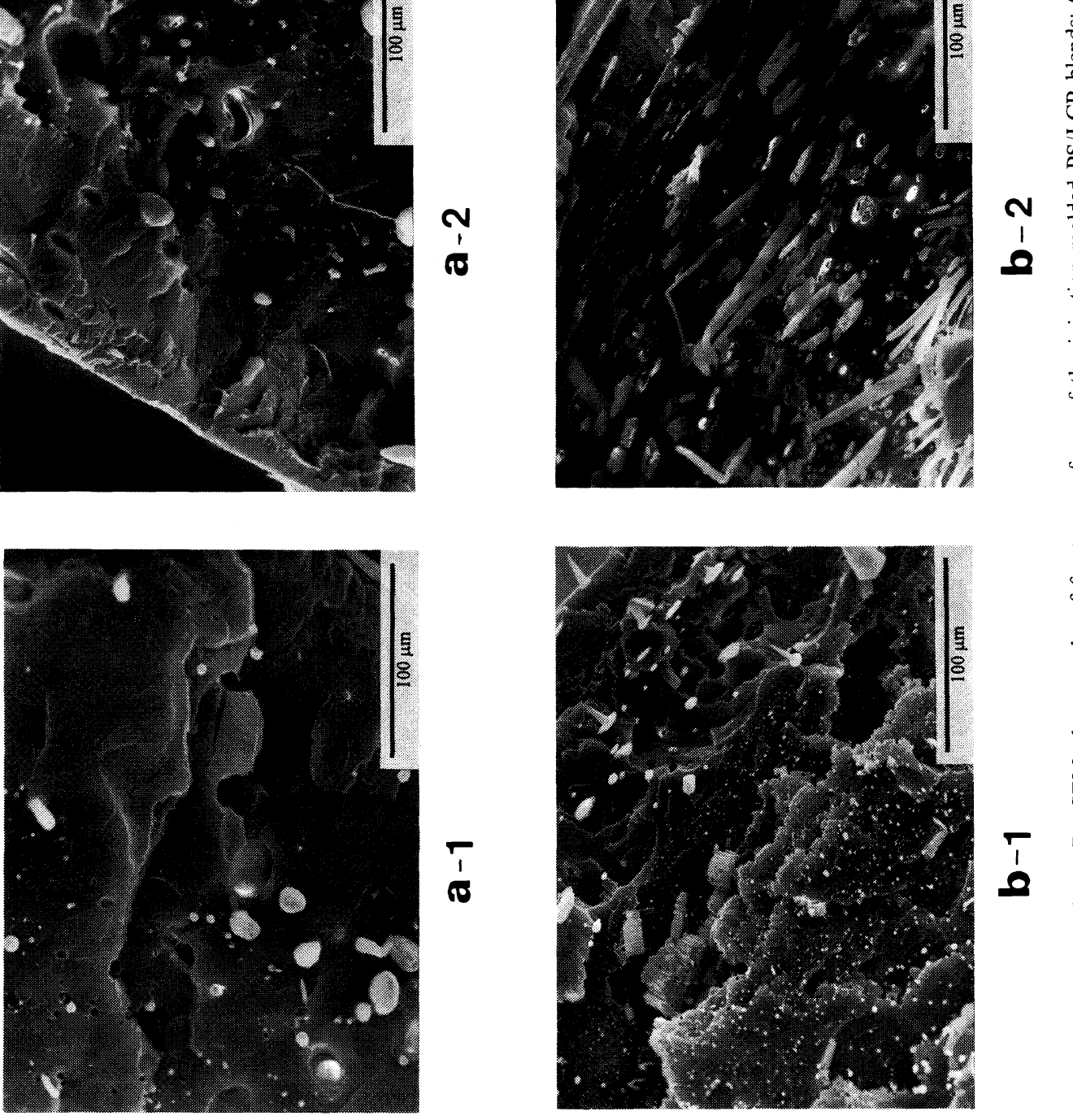

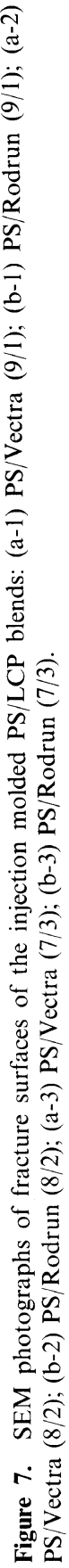



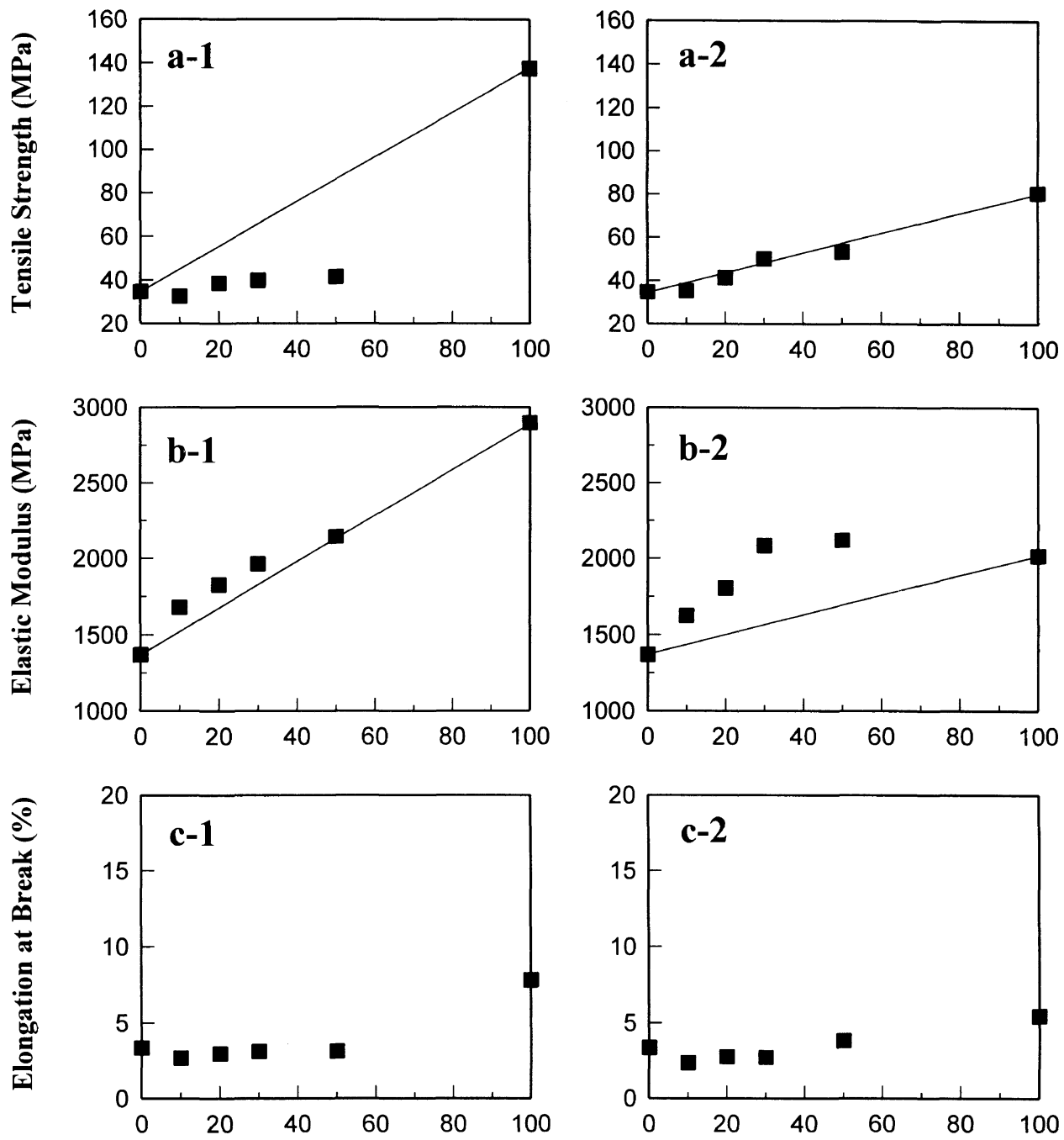

Blend Ratio (wt\% LCP)

Blend Ratio (wt\% LCP)

Figure 8. Mechanical properties of the PS/LCP blends. The figures of a-1, b-1, and c-1 represent for PS/Vectra blends and the figures of a-2, b-2, and $\mathrm{c}-2$ represent for PS/Rodrun blends, respectively. The straight lines in figures represent the simple additive rule of mixture.

of Rodrun than in the case of Vectra, because the backbone of Rodrun has a flexible chain which may be more compatible with PS.

The upper limit of the modulus of the two-phase system was given by the parallel connection model of both elements. On the other hand, the lower limit of the modulus was given by the series connection model. In the cases of PS/LCP blends, the upper limit of modulus will be a simple additive rule of mixtures. When the contribution from the dispersed LCP phase was low, the blends showing lower modulus than the linear relationship were evaluated as "poor". On the other hand, the blends showing higher modulus than the linear relationship were evaluated as "excellent".

Figure 8(b) shows the elastic modulus of LCP blends as a function of LCP content. The elastic modulus was improved with the increase of LCP content for both the PS/Vectra blends and PS/Rodrun blends. Moreover, the modulus of the PS/Rodrun blends show a strong positive derivation from simple additive rule of mixtures. These results were consistent with the morphological observations, which showed a better fibrillation in PS/Rodrun blends than in PS/Vectra blends (see Figure 7). For more than $30 \mathrm{wt} \%$ LCP in PS/Rodrun blends, the elastic modulus of the blend was higher than the value of the pure Rodrun LCP. This increase in stiffness may arise from the good orientation of the well developed fibrillar structure of LCP phase in the PS matrix, as shown in Figure 7(b-3). Elongation at break as a function of composition is shown in Figure 8(c). Samples with low contents of LCP show a shallow minimum in the elongation at break, but almost equal values over the entire composition because of the brittle fracture manner of PS thermoplastic matrix. This low elongation at break of LCP blends is typical for LCP and their blends, since the thermoplastic matrix is reinforced with a brittle LCP component. In summary, the PS/Rodrun blends give better mechanical properties than the PS/Vectra blends due to better fibril formation.

\section{CONCLUSIONS}

The effects of viscosity ratio of dispersed phase to matrix on the morphological, rheological, and mechanical properties of blends of PS and two rheologically difflerent LCPs (Vectra and Rodrun) were investigated. 
When the viscosity of the dispersed LCP is higher than that of the thermoplastic matrix polymer as observed in PS/Vectra blends, the viscosity-composition curve shows a minimum at about $10 \mathrm{wt} \%$ of the LCP content. However, when the viscosity of LCP is lower than that of the thermoplastic matrix as observed in PS/Rodrun blends, the viscosity of blends lies between those of the two components.

The microstructures of fracture surface of LCP blends show that Rodrun LCP phases show the fine fibrillar structures in the PS/Rodrun blends and appear to be distributed uniformly in PS matrix, whereas dispersed Vectra LCP phases in the PS/Vectra blends show the spherical form of LCP domains and exhibit less oriented structures. Mechanical properties of injection molded specimens show that the modulus of LCP blends show a positive deviation from the additive rule when the viscosity of the dispersed LCP phase is lower than that of the matrix phase as observed in PS/Rodrun blends. These mechanical behaviors are consistent with the morphological characteristics of the PS/LCP blends. In summary, the viscosity ratio of the dispersed phase to the matrix is an important factor in determining the morphology which in turn determines the mechanical properties of LCP blends.

Acknowledgments. The authors thank the Ministry of Trade, Industry and Energy of the Rebublic of Korea for their financial support (1991).

\section{REFERENCES}

1. D. R. Paul and S. Newman, Ed., "Polymer Blends," Academic Press, New York, N.Y., 1978.

2. D. R. Paul and L. H. Sperling, Ed., "Multicomponent Polymer
Materials," The American Chemical Society, Washington, D.C., 1986.

3. G. Kiss, Polym. Eng. Sci., 27, 410 (1987).

4. K. G. Blizard and D. G. Baird, Polym. Eng. Sci., 27, 653 (1987).

5. G. V. Laivins, Macromolecules, 22, 3974 (1989).

6. T. Sun, D. G. Baird, H. H. Huang, D. S. Done, and G. L. Wilkes, J. Compt. Mater., 25, 788 (1991).

7. C. U. Ko, G. L. Wilkes, and C. P. Wong, J. Appl. Polym. Sci., 37, 3063 (1989).

8. D. Beery, S. Kenig, and A. Siegmann, Polym. Eng. Sci., 31, 451 (1991).

9. W. H. Jo, H. Yim, I. H. Kwon, and T. W. Son, Polym. J., 24, 519 (1992).

10. T. Limtasiri and A. I. Isayev, J. Appl. Polym. Sci., 42, 2923 (1991).

11. G. Crevecoeur and G. Groeninckx, Polym. Eng. Sci., 30, 532 (1990).

12. A. Apicella, P. Iannelli, L. Nicodemo, L. Nicolais, A. Roviello, and A. Sirigu, Polym. Eng. Sci., 26, 600 (1986).

13. J. V. Seppala, M. T. Heino, and C. Kapanen, J. Appl. Polym. Sci., 44, 1051 (1992).

14. M. T. Heino and J. V. Seppala, J. Appl. Polym. Sci., 44, 2185 (1992).

15. M. J. Folkes, "Short Fiber Reinforced Thermoplastics," John Wiley and Sons, New York, N.Y., 1982.

16. R. A. Weiss, W. S. Huh, and L. Nicolais, Polym. Eng. Sci., 27, 684 (1987).

17. S. H. Kim, G. D. Choi, and W. H. Jo, Polym. J., 27, 693 (1995)

18. A. Kohli, N. Chung, and R. A. Weiss, Polym. Eng. Sci., 29, 573 (1989).

19. A. Siegmann, A. Dagan, and S. Kenig, Polymer, 26, 1325 (1985).

20. G. D. Choi, W. H. Jo, and H. G. Kim, J. Appl. Polym. Sci., in press.

21. M. T. Heino, P. T. Hietaoja, T. P. Vainio, and J. V. Seppala, J. Appl. Polym. Sci., 51, 259 (1994).

22. S. M. Hong and B. C. Kim, Polym. Eng. Sci., 34, 1605 (1994).

23. G. D. Choi, S. H. Kim, W. H. Jo, and M. S. Rhim, J. Appl. Polym. Sci., 55, 561 (1995).

24. K. Min, J. L. White, and J. F. Fellers, Polym. Eng. Sci., 24, 1327 (1984).

25. B. R. Bassett and A. F. Yee, Polym. Composites, 11, 10 (1990).

26. F. P. La Mantia and A. Varlenza, Makromol. Chem., Makromol. Symp., 56, 151 (1992). 